Association for Information Systems AIS Electronic Library (AISeL)

AMCIS 1998 Proceedings

Americas Conference on Information Systems

(AMCIS)

December 1998

\title{
Designing End-User Geographic Information Systems
}

Lawrence West

Jr. University of Central Florida

Follow this and additional works at: http://aisel.aisnet.org/amcis 1998

\section{Recommended Citation}

West, Lawrence, "Designing End-User Geographic Information Systems" (1998). AMCIS 1998 Proceedings. 137.

http://aisel.aisnet.org/amcis1998/137

This material is brought to you by the Americas Conference on Information Systems (AMCIS) at AIS Electronic Library (AISeL). It has been accepted for inclusion in AMCIS 1998 Proceedings by an authorized administrator of AIS Electronic Library (AISeL). For more information, please contact elibrary@aisnet.org. 


\title{
Designing End-User Geographic Information Systems
}

\author{
Lawrence A. West, Jr. \\ Department of Management \\ College of Business \\ University of Central Florida
}

\begin{abstract}
Geographic information systems are becoming more popular for end-user and decision support system construction but they incorporate software and concepts with some inherent problems for end-user computing. This paper identifies several concepts developed during the construction of one end-user GIS that can be incorporated in a wide range of end-user GIS. The approaches make extensive use of metadata storage and may include the use of specialized software tools to reduce the burden of system operation and improve data integrity.
\end{abstract}

\section{Introduction}

Geographic information systems (GIS) are a specialized class of database management systems that allow users to analyze, relate, and display spatial attributes of data in addition to conventional relational data. As with many information technologies, however, presenting these capabilities to end-users for ad hoc system development, direct data manipulation, or decision support, requires specialized planning to reduce user effort, protect vital data, and reduce costs.

This paper presents design techniques derived from the successful implementation of an end-user GIS for managing Florida's marine resources (FMRIS). These techniques can serve as a guide for new fielding efforts and help database administrators manage the tradeoffs between user access and flexibility on one hand and security and cost avoidance. The techniques have already worked in one GIS-based system and can be incorporated in other designs.

\section{Common Themes}

This section previews some common themes that run through the detailed discussions of problem mitigation that follow.

As with large conventional database systems the most important theme is the use of metadata about the spatial coverages (Bedoll \& Kimball 1990). All but one of the remedial efforts discussed below relies on stored data about the individual GIS coverages.

Second, the use of metadata requires an extensive coverage and database documentation effort. While such efforts are assumed for a well-designed conventional organizational database the author has found that the majority of GIS data sets are not created by MIS professionals. One of the most striking results of this tendency is the lack of formal documentation standards.

Third, the remedial measures that follow can be implemented in a number of ways. In the FMRIS example they were implemented in an event- and menu-driven interface that forced their use on the user. It is entirely possible to provide these same capabilities via stand-alone tools that the user can choose to use or not, depending on the nature of the task and the user's expertise.

\section{Five User Problems}

The following sections discuss five end-user problems specific to the use of GIS systems along with the mitigating techniques developed for FMRIS.

\section{Identifying Relevant Coverages}

This GIS-specific issue is analogous to the problem of selecting tables and fields for use in a conventional database management system. As with a conventional system, the user's familiarity with the data, its organization, and content, is critical for successful unchauffeured access (Rockart \& Flannery 1983). The FMRIS experience identified three classes of user actions resulting in the need to load or use one or more specific geographic coverages: accessing a specific coverage directly, accessing a specific coverage based on its contents, and accessing a specific coverage as part of a query.

Accessing a Specific Coverage: Many times the user will want to work with a specific coverage but there are two problems with this task. First, many GIS systems store coverages in directories rather than individual files or named tables within a comprehensive DBMS. Second, naming schemes for GIS coverages tend to be haphazard. Many individual GIS systems incorporate coverages created by a number of different organizations. Externally acquired coverages will have naming schemes that typically must apply to a number of operating systems and that have no inherent relationship to the organization's internally produced naming schemes. 
The solution to this problem presents the first use of the previously discussed metadata. FMRIS used a single table called Coverages that stored a great deal of information about the individual coverage. Four fields in particular, Name, Description, FileName, and Path, are used to overcome these problems. Name stores a 20 character name for the coverage that is significantly more meaningful than the very cryptic names that came with the imported coverages. Description stores a 256 character description of the coverage's contents. Whenever users select a coverage by name they only saw a menu of the Name fields, not the stored file name (actually the directory name). In FMRIS, whenever users highlight an individual Name entry in a list the corresponding Description field was displayed in a help box on the input form before the user committed to the selection. When the user selects a particular coverage the Path and FileName fields are used to tell the system where to find the coverage. This same approach can be easily implemented in any GIS coverage intended for end-user use.

Accessing a Coverage by Content: As with many conventional DBMS the user may want to load coverages based on their contents or applicability without knowing exactly what coverages would be suitable in a given situation.

In FMRIS the SiteType, CoverageSiteType, and Coverage tables provided critical information to allow users to load coverages based on content. The SiteType table lists key concepts of interest to users of the system. The SiteType table also contains a lengthier description of each concept of interest for use in the user interface.

Because the CoverageSiteType table links concepts with coverages the user can select concepts of interest from the SiteType table. The system uses the linking table and the Path and FileName attributes of the Coverage table to determine which coverages to load. Again, the same cataloging feature can be provided to users

Accessing Tables Through Queries: Many user actions in a comprehensive GIS require linking GIS coverages to other coverages or to 'conventional' tables in a DBMS. In FMRIS linkages were universally accomplished with stored queries which may or may not allow the user to specify parameters for a Where clause through a GUI interface. Queries were embedded in either the procedural code and any user selections were concatenated into the Where clause before the query was executed.

\section{Layering Coverages}

While the ability to create spatial joins between geographic coverages provides an important data analysis capability in GIS (West \& Mennecke 1998), some of these systems' strongest contributions come from their ability to present visual relationships between geographic objects (Crossland, et al. 1995; Grupe 1990). Because spatial coverages can include dense or less dense polygon, line, and point coverages it is important for coverages to 'layer' appropriately so as to avoid, to the extent possible, masking less dense coverages with denser ones.

FMRIS supported users in this task by storing information about the density of the objects in a coverage. The Level field Coverage table allows the person mounting the software to indicate the relative density of the features in the coverage. When coverages are loaded by the system a recordset of desired coverage path and file names is created using an Order By clause on the Level field. Coverages are then loaded in the order of the dynaset, automatically placing the most dense coverages on the bottom of the 'stack.'

\section{Constructing Spatial Joins}

Spatial joins and selections enable the construction of queries in which a geographic selection of records based on their locations also leads to the selection of attribute records for the selected objects.

Some end-user GIS provide dialogs for performing spatial selections and joins. In these cases, and when constructing systems for command level users (Rockart \& Flannery 1983), supporting users with identification and loading of appropriate themes may be sufficient. When constructing systems for nonprogramming end-users, however, additional assistance may be required.

In FMRIS the spatial selections were accomplished by anticipating the tasks users would perform and embedding the selection syntax in code. Users can easily select the coverages to be selected and the system constructs and executes the spatial selection based on user input. Similar techniques can be embedded in comprehensive systems or can be scripted as an aide for command level users.

\section{Editing/Adding Data}

Data integrity is an important concern in any end-user computing environment (Alavi \& Weiss 1985; Henderson \& Treacy 1986; O'Donnell \& March 1987) and end-user GIS pose some special integrity problems. First, command level use of GIS data through GIS software provides generally unfettered access to the underlying data, including spatial data. Whereas modern database management systems provide many features for enforcing data integrity these features are generally lacking in GIS software. Changing shape data can be especially insidious as users may become used to manipulating shapes drawn on the screen and will may not realize that they are now changing the locations, vertices, or sizes of shapes from a coverage.

There are four approaches that can be taken to help manage data integrity in an end-user GIS environment:

Using Copies of Critical Data: One of the simplest solutions to the data integrity problem is to provide end-users with copies of critical data. They cannot affect the main data which can be both an advantage and a disadvantage.

Using Advanced GIS Systems: Some companies are now producing GIS extensions to modern relational database management systems (RDBMS) such as Oracle and DB2. In these systems the attribute data is stored in the RDBMS and the shape data is stored in special storage structures. Of particular interest is that the tools available for enforcing data integrity in the RDBMS can apply to the attribute portion of the GIS data. 
Unfortunately, these systems still do not enforce integrity constraints on the shape data. West and Mennecke identified several GIS-specific data integrity rules that should be managed in a complete system (West \& Mennecke 1998).

Restricting User Access: In FMRIS users required a special login password to have access to data editing features. When other classes of users logged on the data editing commands for both attribute and spatial data were disabled.

Specialized Integrity Checkers: It is possible to construct specialized data integrity checkers that do not rely on stored constraints in the RDBMS. In FMRIS, for example, when users added attribute data extensive domain integrity checks were performed before the data was posted. Enforcing data integrity rules for spatial data is more problematic and has not, to the author's knowledge, been implemented yet. West and Mennecke developed rules for specifying spatial integrity (1998) and these rules are not, in principle, difficult to enforce.

\section{Reporting Results}

The final category of user support is in the area of preparing map results of a GIS analysis. While GIS are designed to construct maps as an integral part of their operations there are nuances to this process that can escape the untrained user. Several elements produce an effective map:

Labeling Map Objects: GIS software has the ability to label objects visible on the map by using the contents of a field in the coverage's attribute data as a label. FMRIS specifies a label field for each coverage as part of the mounting process so there is no guesswork involved in selecting a field.

Controlling Display Scale: Many GIS allow the user to specify the minimum and maximum scale at which a coverage will display. This capability gives the effect of automatically turning coverages on or off as the display zooms in or out. Unfortunately, determining appropriate display scales requires an understanding of the significance of scale that may be difficult for inexperienced users to grasp. The FMRIS mounting process allows the expert to specify a minimum and maximum display resolution. These values are then stored in the fields minRes and maxRes in the Coverage table. The GIS then automatically turns coverages on or off as the user zooms in or out with the map.

Establishing a Color Scheme: Color schemes are important elements of map design but, unfortunately, most GIS do not store color schemes with the coverages. FMRIS uses the metadata at two different levels to overcome this burden. Both individual coverages and specific field values can have customized colors assigned and these colors are applied whenever a coverage is loaded.

Placing Map Objects: The final topic covers the construction and placing of peripheral map elements. FMRIS automates the construction of a scale for the map, a North arrow, and an inset map. The scale and north arrow are automatically placed in the marginal area of the map while the FMRIS gives the user the ability to specify the location of the inset map (if one is desired at all).

While these three objects may not be the most important aspects of user support it turns out that they are also not intuitive to create with some software packages. By automating their creation and placement FMRIS provides one more level of support to increase the usability of the GIS software while reducing the burden on the user.

\section{Conclusions}

GIS are powerful information management systems with particular suitability for decision support and end-user applications. However, their complexity, their use of complex data, and the need for applying obscure cartographic principles makes their immediate use by end-users problematic. On the other hand, many of the GIS-specific problems for end-users can be mitigated or overcome through the implementation of electronic assistants to perform many of these tasks. These mitigating steps can be implemented as part of a comprehensive application for end-users as in the case of FMRIS or can be created as independent applications for use in more free-form end-user systems.

One of the key findings of the FMRIS experience is the importance of the role of metadata in supporting end-users. FMRIS, as well as the specific approaches recommended here, require that an expert provide key descriptive information about coverages when they are added to the database. This one-time overhead step then provides essential information used by the user-support components. Fortunately, the idea of a metadata repository is not at odds with conventional views of user support for data access. The geographic elements of an end-user GIS just increases the complexity of the mounting process slightly.

Next, the construction of the assistance agents also requires additional overhead. A key decision, then, is whether to provide end-user support through an investment in software agents or through user training. While recommending an approach to this decision is beyond the scope of this paper it is clear that the capital vs. labor investment decision is familiar to many managers and offers no particular difficulties, at least as long as the software investments are well understood. It is hoped that this paper contributes to this end.

Finally, there is a rich literature on the support, management, and control of end-user computing in modern organizations, much of which was not specifically addressed in this paper. It is important to realize that much of this literature can apply to GIS if the relevant criteria are met. This focus has focused on the GIS-specific aspects of end-user support. It is felt that addressing these issues can significantly increase the use, and therefore the value, of an organization's investment in GIS technology.

References

References and an expanded version of this paper are available from the author (lwest@bus.ucf.edu). 